\title{
Article
}

\section{Ground reaction forces of elite dressage horses in collected trot and 1 passage}

Clayton, Hilary M., Schamhardt, Henk C. and Hobbs, Sarah Jane Available at http://clok.uclan.ac.uk/16965/

Clayton, Hilary M., Schamhardt, Henk C. and Hobbs, Sarah Jane ORCID: 00000002-1552-8647 (2017) Ground reaction forces of elite dressage horses in collected trot and 1 passage. The Veterinary Journal, 221 . pp. 30-33. ISSN 1090-0233

It is advisable to refer to the publisher's version if you intend to cite from the work. http://dx.doi.org/10.1016/j.tvjl.2017.01.016

For more information about UCLan's research in this area go to http://www.uclan.ac.uk/researchgroups/ and search for < name of research Group>.

For information about Research generally at UCLan please go to http://www.uclan.ac.uk/research/

All outputs in CLoK are protected by Intellectual Property Rights law, including Copyright law. Copyright, IPR and Moral Rights for the works on this site are retained by the individual authors and/or other copyright owners. Terms and conditions for use of this material are defined in the policies page.

\section{CLoK}

Central Lancashire online Knowledge www.clok.uclan.ac.uk

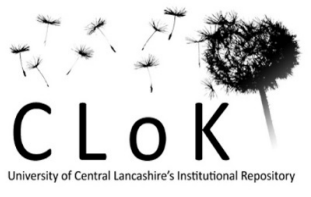




\section{Ground reaction forces of elite dressage horses in collected trot and passage}

*Hilary M. Clayton ${ }^{\mathrm{a}}$, Henk C. Schamhardt ${ }^{\mathrm{b}, \dagger}$ and Sarah Jane Hobbs ${ }^{\mathrm{c}}$

Mary Anne McPhail Equine Performance Center, Michigan State University, 736 Wilson Road, East Lansing, MI 48824, USA

${ }^{a}$ Sport Horse Science, 3145 Sandhill Road, Mason, MI 48854, USA

Email: claytonh@cvm.msu.edu

${ }^{b}$ Faculty of Veterinary Medicine, Utrecht University, Utrecht, The Netherlands

${ }^{\dagger}$ Deceased

${ }^{c}$ University of Central Lancashire, Centre for Applied Sport and Exercise Sciences, Preston, UK

*Corresponding author: Hilary M. Clayton

Email: claytonh@cvm.msu.edu

Phone/fax: 517-333-3833 


\begin{abstract}
This study compares sagittal plane ground reaction forces (GRF) in ridden dressage horses performing collected trot and passage overground. In-ground force plates captured GRF data from eight elite dressage horses, four Dutch Warmbloods and four Lusitanos, ridden by their own trainers. At least three stance phases were analysed for forelimbs and hindlimbs per horse. The variables extracted were vertical and longitudinal (braking, propulsive) force maxima, their times of occurrence and the respective impulses for forelimbs and hindlimbs. Lusitanos had lower vertical impulses than Dutch warmbloods in collected trot. Across all horses, passage had larger vertical impulses than collected trot in forelimbs and hindlimbs. Propulsive impulse increased in the hindlimbs in passage. Prolonged stance durations in passage contributed to higher vertical impulses that are needed to increase the vertical excursions of the centre of mass (COM).
\end{abstract}

Keywords: Dressage; Collection; Kinetics; Dutch Warmblood; Lusitano 
Dressage horses maintain an uphill longitudinal balance by adjusting limb protraction/retraction and ground reaction forces (GRF) (Hobbs and Clayton, 2013). For collected trot and passage (Fédération Equestre Internationale, 2016) vertical GRFs have been compared in horses ridden on a treadmill (Weishaupt et al., 2009), but treadmill locomotion differs from overground physiologically and kinematically (Barrey et al., 1993; Sloet van Oldruitenberg-Oosterbaan and Barneveld, 1995). This study advances knowledge by comparing vertical and longitudinal GRFs and impulses of collected trot and passage in elite dressage horses ridden overground. The experimental hypotheses are that in passage both forelimbs and hindlimbs generate higher vertical impulses to provide greater vertical oscillation of the COM and lower longitudinal impulses to align the GRF vector with the smaller ranges of limb protraction/retraction.

The protocol was approved by the institutional animal care and use committee. Eight sound, Grand Prix dressage horses were ridden by their regular trainers. Four Dutch Warmbloods (weight: 550-745 kg) were warmed up then ridden along a $20 \mathrm{~m}$ rubberized runway with an embedded force plate (Type Z4852C, Kistler Corporation, $300 \mathrm{~Hz}$ ). Four Lusitanos (weight: $597-613 \mathrm{~kg}$ ) were warmed up and ridden along a $30 \mathrm{~m}$ rubberized runway with four embedded force plates (FP60120 and FP6090, Bertec Corporation, $960 \mathrm{~Hz}$ ).

Trials of collected trot and passage were recorded in a predetermined random order. Trials assessed as inadequate by the riders or an experienced observer were discarded. In successful trials the horse moved straight at consistent velocity making one or more valid force plate contacts. At 
least three stance phases for a forelimb and hindlimb were analyzed per horse/gait. Since trot and passage are symmetrical gaits, left and right limbs were grouped (Weishaupt et al., 2009). GRFs were normalized by dividing the forces by the combined mass of horse and rider. Variables derived from the GRFs were stance duration (threshold value $50 \mathrm{~N}$ for contact and lift off); peak values and times of occurrence of vertical, longitudinal braking and longitudinal propulsive GRFs and their respective impulses, and time of zero longitudinal force.

A Kolmogorov Smirnov test indicated that variables were normally distributed except times to peak hindlimb vertical GRF and peak forelimb propulsive GRF. Independent samples ttests compared values for the two breeds and data were then combined. Repeated measures ANOVA (normally distributed) or the Wilcoxon signed rank test (non-normally distributed) was used for between-gait (collected trot, passage) and between-limb (fore, hind) comparisons.

Two variables differed significantly between breeds. In collected trot but not passage, Dutch Warmbloods had higher vertical impulses in the forelimbs (collected trot: Warmbloods $2.57 \pm 0.13$, Lusitanos $2.21 \pm 0.02 \mathrm{Ns} / \mathrm{kg}, P=0.006$, passage: Warmbloods $2.85 \pm 0.07$, Lusitanos $2.81 \pm 0.15 \mathrm{Ns} / \mathrm{kg}, P=0.593$ ) and hindlimbs (collected trot: Warmbloods $1.90 \pm 0.08$, Lusitanos $1.64 \pm 0.02 \mathrm{Ns} / \mathrm{kg}, P=0.003$, passage: Warmbloods $2.49 \pm 0.11$, Lusitanos $2.31 \pm 0.07 \mathrm{Ns} / \mathrm{kg}$, $P=0.069$ ). Forelimb and hindlimb stance durations were longer for passage. Peak vertical force did not differ but the longer stance duration resulted in significantly higher vertical impulses in all limbs in passage (Table 1, Fig. 1). Hindlimb peak propulsive force occurred relatively 
(percent stance) earlier and was accompanied by a large and significant increase in propulsive impulse in passage due to both the prolonged stance duration and earlier transition from braking to propulsion (Table 1, Fig. 2). Comparing forelimbs vs hindlimbs, all variables differed significantly $(P<.05)$ except peak vertical force and its time of occurrence during passage.

The coordinated fore- and hindlimb GRFs provide gravitational support together with inertial forces to accelerate the $\mathrm{COM}$, maintain forward progression, and control trunk orientation (Hobbs and Clayton, 2013). Detailed knowledge of GRFs is needed to evaluate the musculoskeletal effects of postural modifications shown by dressage horses performing highly collected movements. In accordance with our first hypothesis, all four limbs contributed significantly to the higher vertical impulse in passage compared with collected trot but the increase was relatively greater in the hindlimbs $(+32 \%)$ than the forelimbs $(+17 \%)$, which is similar to the increases (forelimbs: $+24.8 \%$; hindlimbs: $+39.9 \%$ ) reported by Weishaupt et al. (2009). The smaller vertical impulses in the Lusitanos at trot were associated with smaller COM vertical oscillations (unpublished data) which may explain why riders find Iberian horses easy to sit at trot.

The summed GRF of all grounded limbs is represented by a vector acting at the centre of pressure (COP). During trotting, the COP initially corresponds with the first hoof to contact the ground. It remains almost stationary through most of diagonal stance in a position closer to the forelimb which has a higher vertical GRF than the hindlimb. In terminal stance, the COP moves toward the fore hoof, which is the last limb to lift off (Hobbs and Clayton, 2013). The COM moves forward continuously during trotting at fairly constant speed relative to the diagonal base 
of support (Hobbs and Clayton, 2013). On a treadmill, the forelimbs provided $57.4 \%$ of the vertical impulse in collected trot and $54.0 \%$ in passage, which was interpreted as moving the COM closer to the hindlimbs in passage (Weishaupt et al., 2009). In the present study, the decrease in forelimb impulse contribution and the resulting change in fore:hind vertical GRF ratio, was interpreted as shifting the COP (not the COM) towards the hindlimbs in passage. Changes in pro-retraction angles of the supporting limbs and their position relative to the COM determine the moment arm lengths of the fore- and hindlimb vertical GRFs (Hobbs and Clayton, 2013).

The hypothesis that passage would have a lower longitudinal impulse associated with reduced limb protraction-retraction was not supported. The higher hindlimb propulsive impulse in passage could contribute to a nose-up moment around the COM that would lift the forehand (Hobbs et al., 2016). In passage the forelimb exerts a braking force while the hindlimb simultaneously exerts a propulsive longitudinal force through much of stance (Figure 2), which results in a marked convergence of their sagittal plane GRF vectors (Figure 3).

It is concluded that increased vertical oscillations of the COM in passage are associated with larger vertical impulses especially in the hindlimbs. The increased hindlimb propulsive impulse may be a balancing strategy to increase the nose-up moment around the COM.

\section{Acknowledgements}


This study was supported by the McPhail endowment at Michigan State University.

\section{Conflict of Interest}

None of the authors of this paper have a financial or personal relationship with other people or organisations that could inappropriately influence or bias the content of the paper.

\section{References}

Barrey, E., Gallous, P., Vallette, J.P., Auvinet, F. and Wolter, R. 1993. Stride characteristics of overground versus treadmill locomotion in the saddle horse. Acta Anatomica 146, 90-94.

Fédération Equestre Internationale. 2016. FEI Dressage Rules. Lausanne, Switzerland. pp 13, 22.

Hobbs, S.J. and Clayton, H.M. 2013. Sagittal plane ground reaction forces, center of pressure and center of mass in trotting horses. The Veterinary Journal 198, e14-e19.

Hobbs, S.J., Bertram, J. and Clayton, H.M. 2016. An exploration of the influence of diagonal dissociation and moderate changes in speed on locomotor parameters in trotting horses. PeerJ 4:e2190 https://doi.org/10.7717/peerj.2190.

Sloet van Oldruitenborgh-Oosterbaan, M.M. and Barneveld, A. 1995. Comparison of the workload of Dutch Warmblood horses ridden normally and on a treadmill. The Veterinary Record 137, 136-139.

Weishaupt, M.A., Byström, A., von Peinen, K., Wiestner, T., Meyer, H., Waldern, N., Johnston, C., Van Weeren, R. and Roepstorff, L. 2009. Kinetics and kinematics of the passage. Equine Veterinary Journal 41, 263-267. 


\begin{tabular}{|l|c|c|c|c|}
\hline \multirow{2}{*}{} & \multicolumn{2}{|c|}{ Forelimbs } & \multicolumn{2}{c|}{ Hindlimbs } \\
\cline { 2 - 5 } & $\begin{array}{c}\text { Collected } \\
\text { Trot }\end{array}$ & Passage & $\begin{array}{c}\text { Collected } \\
\text { Trot }\end{array}$ & Passage \\
\hline Peak vertical force & 10.06 & 9.74 & 8.28 & 8.48 \\
(N/kg) & $(0.54)$ & $(1.16)$ & $(0.79)$ & $(1.09)$ \\
\hline Time of peak vertical force & 53.25 & 47.83 & 46.76 & 43.33 \\
(\% stride) & $(3.57)$ & $(4.81)$ & $(1.32)$ & $(4.77)$ \\
\hline Vertical impulse & 2.44 & 2.85 & 1.81 & 2.43 \\
(Ns/kg) & $(0.20)$ & $(0.08)$ & $(0.14)$ & $(1.03)$ \\
\hline Peak braking force & -1.07 & -0.95 & -0.51 & -0.24 \\
(N/kg) & 0.11 & $(0.29)$ & $(0.16)$ & $(0.23)$ \\
\hline Time of peak braking force & 30.10 & 29.02 & 22.34 & 18.22 \\
(\% stride) & $(2.46)$ & $(4.63)$ & $(1.77)$ & $(4.13)$ \\
\hline Braking impulse & -0.16 & -0.16 & -0.05 & -0.03 \\
(Ns/kg) & $(0.03)$ & $(0.06)$ & $(0.02)$ & $(0.03)$ \\
\hline Peak propulsive force & 0.77 & 0.48 & 1.04 & 0.92 \\
(N/kg) & $(0.28)$ & $(0.32)$ & $(0.16)$ & $(0.23)$ \\
\hline Time of peak propulsive force & 82.25 & 84.29 & 70.07 & 64.11 \\
(\% stride) & $(2.88)$ & $(5.73)$ & $(1.35)$ & $(4.17)$ \\
\hline Propulsive impulse & 0.07 & 0.06 & 0.13 & 0.19 \\
(Ns/kg) & $(0.03)$ & $(0.05)$ & $(0.02)$ & $(0.05)$ \\
\hline Time of zero longitudinal force & 60.71 & 64.05 & 39.85 & 21.27 \\
(\% stride) & $(3.48)$ & $(9.16)$ & $(4.08)$ & $(3.92)$ \\
\hline Stance duration & 0.38 & 0.47 & 0.36 & 0.49 \\
(ms) & $(0.03)$ & $(0.04)$ & $(0.03)$ & $(0.08)$ \\
\hline
\end{tabular}

Table 1: Vertical and longitudinal ground reaction forces and stance durations for the forelimbs and hindlimbs of horses $(\mathrm{N}=8)$ performing collected trot and passage. Shaded boxes indicate values that differ significantly between collected trot and passage $(P<0.05)$. Note that values are standardized to the combined body masses of horse and rider (forces) or to time (percent stride). 


\section{Figure Legends}

Figure 1: Vertical ground reaction forces in the hindlimbs (above) and forelimbs (below) for a diagonal stance phase in collected trot (dark line) and passage (light line) in one horse that made synchronized contacts with the fore and hind limbs on separate force plates. Note that absolute values are reported.

Figure 2: Longitudinal ground reaction forces in the hindlimbs (above) and forelimbs (below) for a diagonal stance phase of collected trot (dark line) and passage (light line) in one horse that made synchronized contacts with the fore and hind limbs on separate force plates. Note that absolute values are reported.

Figure 3: Stick figure of horse performing passage. The arrows show convergence of the sagittal plane ground reaction force vectors of the fore- and hindlimbs at the moment of maximal vertical force. 